\title{
A Study of SUOWA Operators in Two Dimensions
}

\author{
Bonifacio Llamazares \\ Departamento de Economía Aplicada, Instituto de Matemáticas (IMUVA), Universidad de Valladolid, \\ Avenida Valle de Esgueva 6, 47011 Valladolid, Spain \\ Correspondence should be addressed to Bonifacio Llamazares; boni@eco.uva.es
}

Received 17 March 2015; Accepted 14 June 2015

Academic Editor: Eckhard Hitzer

Copyright ( $\odot 2015$ Bonifacio Llamazares. This is an open access article distributed under the Creative Commons Attribution License, which permits unrestricted use, distribution, and reproduction in any medium, provided the original work is properly cited.

SUOWA operators are a new class of aggregation functions that simultaneously generalize weighted means and OWA operators. They are Choquet integral-based operators with respect to normalized capacities; therefore, they possess some interesting properties such as continuity, monotonicity, idempotency, compensativeness, and homogeneity of degree 1 . In this paper, we focus on two dimensions and show that any Choquet integral with respect to a normalized capacity can be expressed as a SUOWA operator.

\section{Introduction}

The study of aggregation operators has received special attention in the last years. This is due to the extensive applications of these functions for aggregating information in a wide variety of areas. Two of the best-known aggregation operators are the weighted means and the ordered weighted averaging (OWA) operators (Yager [1]). Both classes of functions are defined by means of weighting vectors, but their behavior is quite different. Weighted means allow weighting each information source in relation to their reliability while OWA operators allow weighting the values according to their ordering.

Although both families of operators allow solving a wide range of problems, both weightings are necessary in some contexts. Some examples of these situations have been given by several authors (see, for instance, Torra [2-4], Torra and Godo [5, pages 160-161], Torra and Narukawa [6, pages 150151], Roy [7], Yager and Alajlan [8], and Llamazares [9] and the references therein) in fields as diverse as robotics, vision, fuzzy logic controllers, constraint satisfaction problems, scheduling, multicriteria aggregation problems, and decision-making.

A typical situation where both weightings are necessary is the following (Llamazares [9]): suppose we have several sensors to measure a physical property. On the one hand, sensors may be of different quality and precision, so a weighted mean type aggregation is necessary. On the other hand, to prevent a faulty sensor from altering the measurement, we might consider an OWA type aggregation where the maximum and minimum values are not taken into account. A similar situation occurs when a committee of experts has to assess several candidates or proposals. On the one hand, a weighted mean type aggregation is suitable for reflecting the expertness or the confidence in the judgment of each expert. On the other hand, an OWA type aggregation allows us to deal with situations where an expert feels excessive acceptance or rejection towards some of the candidates or proposals.

Different aggregation operators have appeared in the literature to deal with this kind of problems. A usual approach is to consider families of functions parameterized by two weighting vectors, one for the weighted mean and the other one for the OWA type aggregation, which generalize weighted means and OWA operators in the following sense. A weighted mean (or an OWA operator) is obtained when the other weighting vector has a "neutral" behavior; that is, it is $(1 / n$, $\ldots, 1 / n$ ) (see Llamazares [10] for an analysis of some functions that generalize the weighted means and the OWA operators in this sense). Two of the solutions having better properties are the weighted OWA (WOWA) operator, proposed by Torra [3], and the semiuninorm based ordered weighted averaging (SUOWA) operator, introduced by Llamazares [9]. 
The good properties of WOWA and SUOWA operators are due to the fact that they are Choquet integral-based operators with respect to normalized capacities. In the case of SUOWA operators, their capacities are the monotonic cover of certain games, which are defined by using the capacities associated with the weighted means and the OWA operators and "assembling" these values through semiuninorms with neutral element $1 / n$.

Because of their good properties, it seems interesting to analyze the behavior of SUOWA operators from different points of view. In this paper, we consider the case of two dimensions that, although simple, is attractive from a theoretical point of view, and we show that any Choquet integral with respect to a normalized capacity can be expressed as a SUOWA operator.

The remainder of the paper is organized as follows. In Section 2 we recall the concepts of semiuninorm and uninorm and give some interesting examples of such functions. Section 3 is devoted to Choquet integral, including some of the most important particular cases: weighted means, OWA operators, and SUOWA operators. In Section 4, we give the main results of the paper. Finally, some concluding remarks are provided in Section 5.

\section{Semiuninorms and Uninorms}

Throughout the paper, we will use the following notation: $N=\{1, \ldots, n\}$; given $A \subseteq N,|A|$ denotes the cardinality of $A$; vectors are denoted in bold and $\boldsymbol{\eta}$ denotes the tuple $(1 / n, \ldots, 1 / n) \in \mathbb{R}^{n}$. We write $\mathbf{x} \geq \mathbf{y}$ if $x_{i} \geq y_{i}$ for all $i \in N$. For a vector $\mathbf{x} \in \mathbb{R}^{n},[\cdot]$ and $(\cdot)$ denote permutations such that $x_{[1]} \geq \cdots \geq x_{[n]}$ and $x_{(1)} \leq \cdots \leq x_{(n)}$.

Semiuninorms are a class of necessary functions in the definition of SUOWA operators. They are monotonic and have a neutral element in the interval $[0,1]$. These functions were introduced by Liu [11] as a generalization of uninorms, which, in turn, were proposed by Yager and Rybalov [12] as a generalization of $t$-norms and $t$-conorms.
Before introducing the concepts of semiuninorm and uninorm, we recall some well-known properties of aggregation functions.

Definition 1. Let $F: \mathbb{R}^{n} \rightarrow \mathbb{R}$ be a function.

(1) $F$ is symmetric if $F\left(x_{\sigma(1)}, \ldots, x_{\sigma(n)}\right)=F\left(x_{1}, \ldots, x_{n}\right)$ for all $\mathbf{x} \in \mathbb{R}^{n}$ and for all permutation $\sigma$ of $N$.

(2) $F$ is monotonic if $\mathbf{x} \geq \mathbf{y}$ implies $F(\mathbf{x}) \geq F(\mathbf{y})$ for all $\mathbf{x}, \mathbf{y} \in \mathbb{R}^{n}$.

(3) $F$ is idempotent if $F(x, \ldots, x)=x$ for all $x \in \mathbb{R}$.

(4) $F$ is compensative (or internal) if $\min (\mathbf{x}) \leq F(\mathbf{x}) \leq$ $\max (\mathbf{x})$ for all $\mathbf{x} \in \mathbb{R}^{n}$.

(5) $F$ is homogeneous of degree 1 (or ratio scale invariant) if $F(r \mathbf{x})=r F(\mathbf{x})$ for all $\mathbf{x} \in \mathbb{R}^{n}$ and for all $r>0$.

Definition 2. Let $U:[0,1]^{2} \rightarrow[0,1]$.

(1) $U$ is a semiuninorm if it is monotonic and possesses a neutral element $e \in[0,1](U(e, x)=U(x, e)=x$ for all $x \in[0,1])$.

(2) $U$ is a uninorm if it is a symmetric and associative $(U(x, U(y, z))=U(U(x, y), z)$ for all $x, y, z \in[0,1])$ semiuninorm.

We denote by $\mathscr{U}^{e}$ (resp., $\mathcal{U}_{i}^{e}$ ) the set of semiuninorms (resp., idempotent semiuninorms) with neutral element $e \in$ $[0,1]$.

SUOWA operators are defined by using semiuninorms with neutral element $1 / n$. Moreover, they have to belong to the following subset (see Llamazares [9]):

$$
\widetilde{\mathscr{U}}^{1 / n}=\left\{U \in \mathcal{U}^{1 / n} \mid U\left(\frac{1}{k}, \frac{1}{k}\right) \leq \frac{1}{k} \forall k \in N\right\} .
$$

Obviously, $\mathcal{U}_{i}^{1 / n} \subseteq \widetilde{\mathscr{U}}^{1 / n}$. Notice that the smallest and the largest elements of $\widetilde{\mathscr{U}}^{1 / n}$ are, respectively, the following semiuninorms:

$$
\begin{aligned}
& U_{\perp}(x, y)= \begin{cases}\max (x, y) & \text { if }(x, y) \in\left[\frac{1}{n}, 1\right]^{2}, \\
0 & \text { if }(x, y) \in\left[0, \frac{1}{n}\right)^{2}, \\
\min (x, y) & \text { otherwise, }\end{cases} \\
& U_{\top}(x, y)= \begin{cases}\frac{1}{k} & \text { if }(x, y) \in I_{k} \backslash I_{k+1}, \text { where } I_{k}=\left(\frac{1}{n}, \frac{1}{k}\right]^{2} \quad(k \in N \backslash\{n\}), \\
\min (x, y) & \text { if }(x, y) \in\left[0, \frac{1}{n}\right]^{2}, \\
\max (x, y) & \text { otherwise. }\end{cases}
\end{aligned}
$$


In the case of idempotent semiuninorms, the smallest and the largest elements of $\mathcal{U}_{i}^{1 / n}$ are, respectively, the following uninorms (which were given by Yager and Rybalov [12]):

$$
\begin{aligned}
& U_{\min }(x, y)= \begin{cases}\max (x, y) & \text { if }(x, y) \in\left[\frac{1}{n}, 1\right]^{2}, \\
\min (x, y) & \text { otherwise, }\end{cases} \\
& U_{\max }(x, y)= \begin{cases}\min (x, y) & \text { if }(x, y) \in\left[0, \frac{1}{n}\right]^{2}, \\
\max (x, y) & \text { otherwise. }\end{cases}
\end{aligned}
$$

In addition to the previous ones, several procedures to construct semiuninorms have been introduced by Llamazares [13]. One of them, which is based on ordinal sums of aggregation operators, allows us to get continuous semiuninorms. Some of the most relevant continuous semiuninorms obtained are the following:

$$
\begin{aligned}
& U_{T_{\mathrm{L}}}(x, y) \\
& = \begin{cases}\max (x, y) & \text { if }(x, y) \in\left[\frac{1}{n}, 1\right]^{2}, \\
\max \left(x+y-\frac{1}{n}, 0\right) & \text { otherwise, }\end{cases} \\
& U_{\widetilde{P}}(x, y)= \begin{cases}\max (x, y) & \text { if }(x, y) \in\left[\frac{1}{n}, 1\right]^{2}, \\
n x y & \text { otherwise, }\end{cases} \\
& U_{T_{\mathrm{M}}}(x, y)= \begin{cases}\max (x, y) & \text { if }(x, y) \in\left[\frac{1}{n}, 1\right]^{2}, \\
\min (x, y) & \text { if }(x, y) \in\left[0, \frac{1}{n}\right)^{2}, \\
x+y-\frac{1}{n} & \text { otherwise, }\end{cases} \\
& U_{P}(x, y)= \begin{cases}\max (x, y) & \text { if }(x, y) \in\left[\frac{1}{n}, 1\right]^{2}, \\
\min (x, y) & \text { if }(x, y) \in\left[0, \frac{1}{n}\right)^{2}, \\
n x y & \text { otherwise. }\end{cases}
\end{aligned}
$$

Notice that the last two semiuninorms are also idempotent. The plots of all these semiuninorms are given, for the case $n=4$, in Figures 1-8.

\section{Choquet Integral}

The notion of Choquet integral is based on that of capacity (see Choquet [14] and Murofushi and Sugeno [15]). The concept of capacity resembles that of probability measure but in the definition of the former additivity is replaced by monotonicity (see also fuzzy measures in Sugeno [16]). A game is then a generalization of a capacity where the monotonicity is no longer required.

Definition 3. (1) A game $v$ on $N$ is a set function, $v: 2^{N} \rightarrow \mathbb{R}$ satisfying $v(\varnothing)=0$.

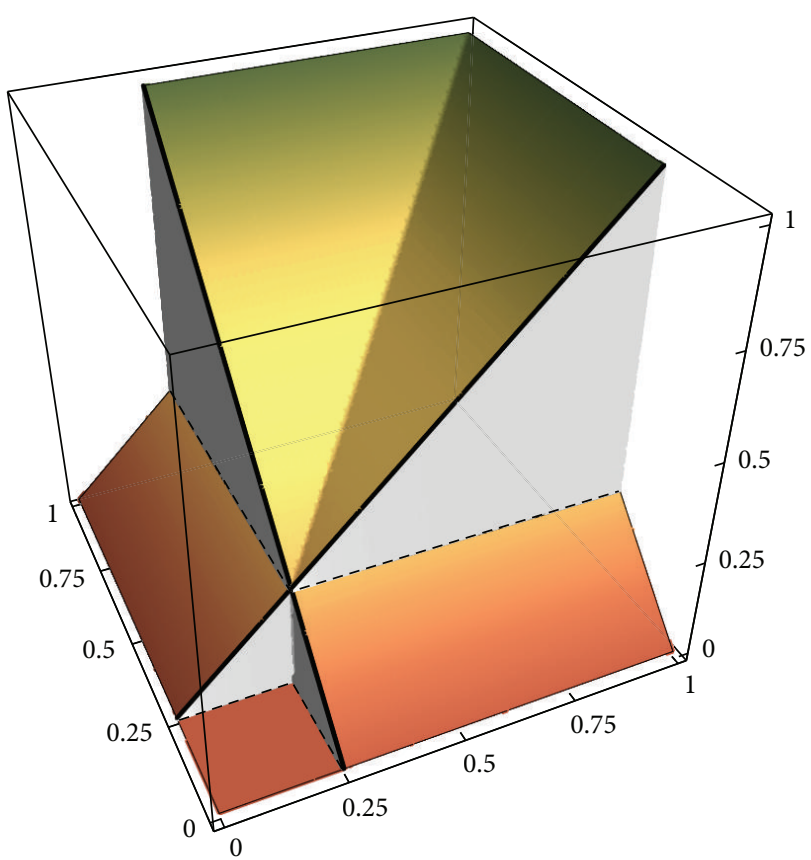

FIgURE 1: The semiuninorm $U_{\perp}$ for $n=4$.

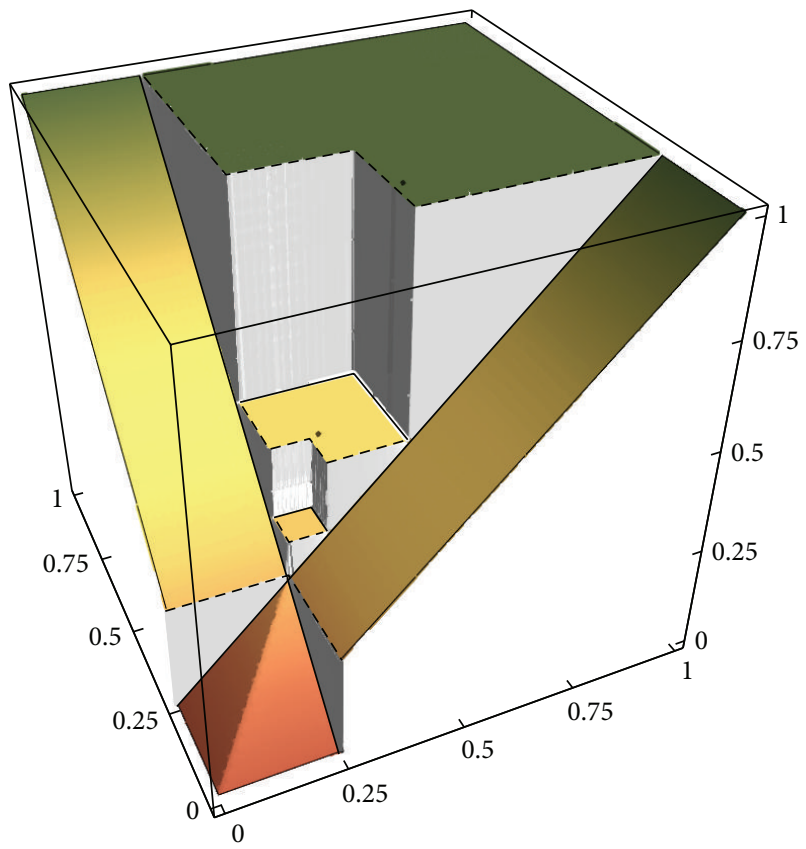

FIgURE 2: The semiuninorm $U_{\mathrm{T}}$ for $n=4$.

(2) A capacity (or fuzzy measure) $\mu$ on $N$ is a game on $N$ satisfying $\mu(A) \leq \mu(B)$ whenever $A \subseteq B$. In particular, it follows that $\mu: 2^{N} \rightarrow[0, \infty)$. A capacity $\mu$ is said to be normalized if $\mu(N)=1$.

A straightforward way to get a capacity from a game is to consider the monotonic cover of the game (see Maschler and Peleg [17] and Maschler et al. [18]). 


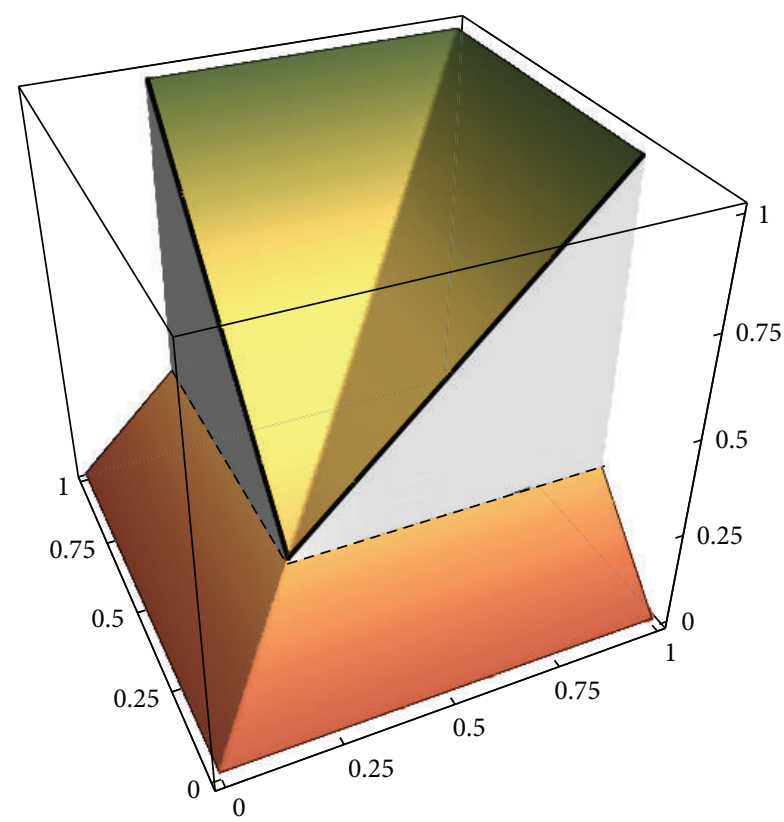

FIgURE 3: The uninorm $U_{\min }$ for $n=4$.

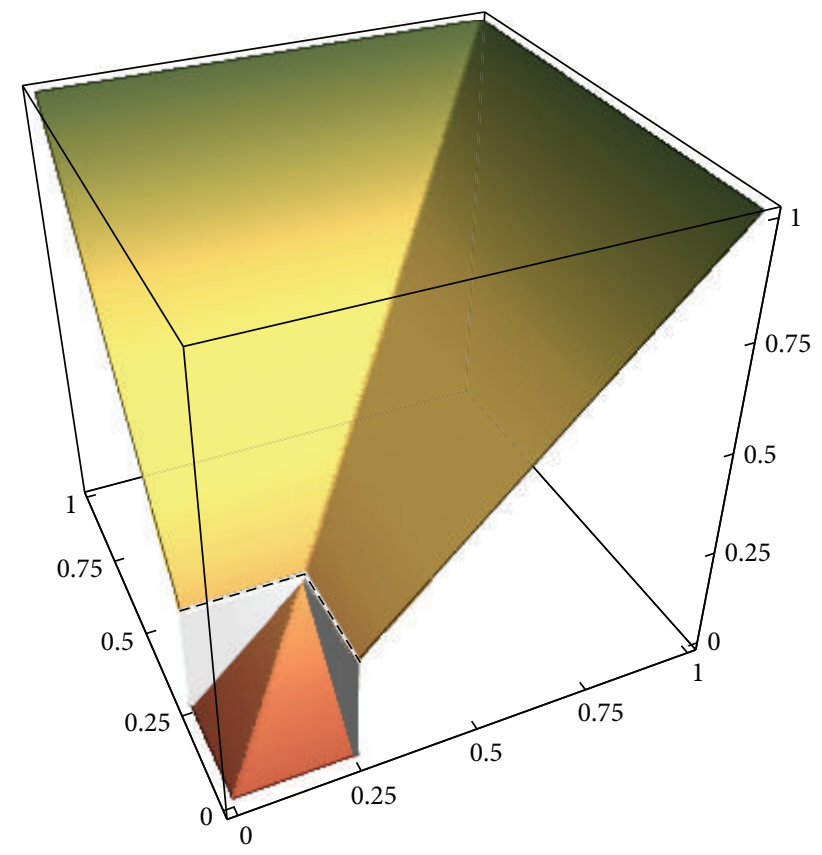

FIgURE 4: The uninorm $U_{\max }$ for $n=4$.

Definition 4. Let $v$ be a game on $N$. The monotonic cover of $v$ is the set function $\widehat{v}$ given by

$$
\widehat{v}(A)=\max _{B \subseteq A} v(B) .
$$

Some basic properties of $\widehat{v}$ are given in the sequel.

Remark 5. Let $v$ be a game on $N$. Then, one has the following:

(1) $\widehat{v}$ is a capacity.

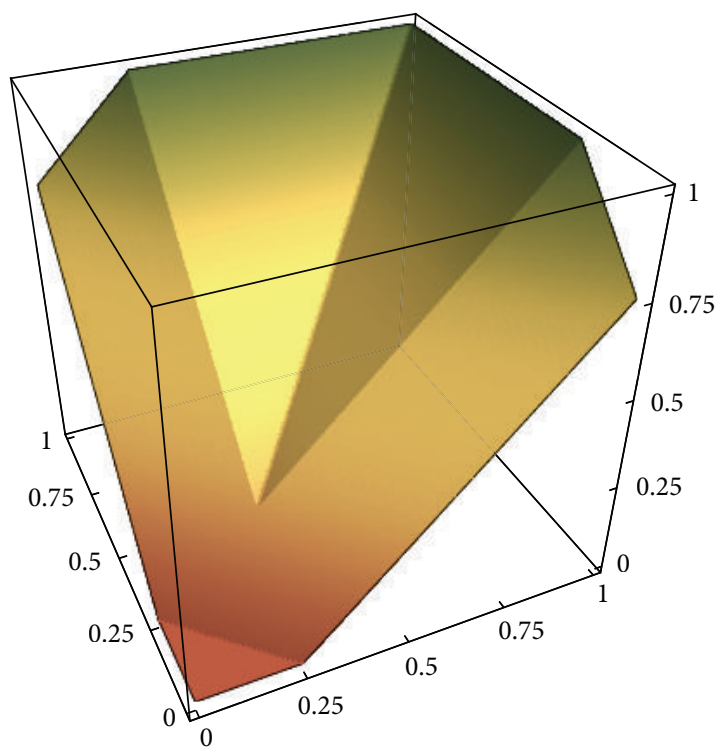

FIgURE 5: The uninorm $U_{T_{\mathbf{L}}}$ for $n=4$.

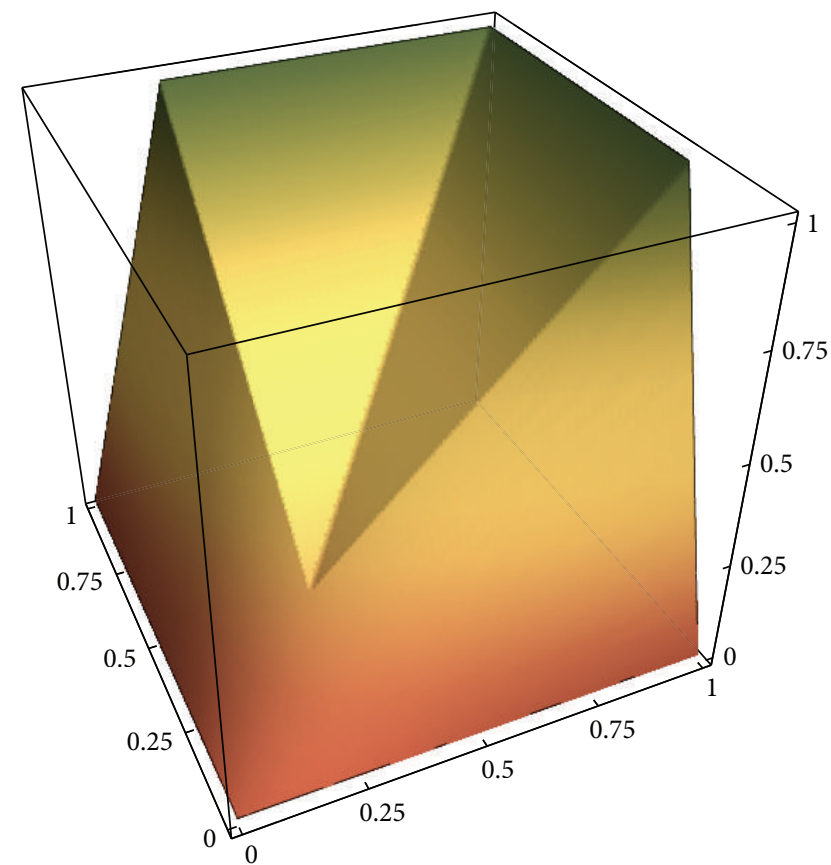

Figure 6: The uninorm $U_{\widetilde{P}}$ for $n=4$.

(2) If $v$ is a capacity, then $\widehat{v}=v$.

(3) If $v(A) \leq 1$ for all $A \subseteq N$ and $v(N)=1$, then $\widehat{v}$ is a normalized capacity.

Although the Choquet integral is usually defined as a functional (see, for instance, Choquet [14], Murofushi and Sugeno [15], and Denneberg [19]), in this paper we consider the Choquet integral as an aggregation function over $\mathbb{R}^{n}$ (see, for instance, Grabisch et al. [20, page 181]). Moreover, we define the Choquet integral for all vectors of $\mathbb{R}^{n}$ instead of 


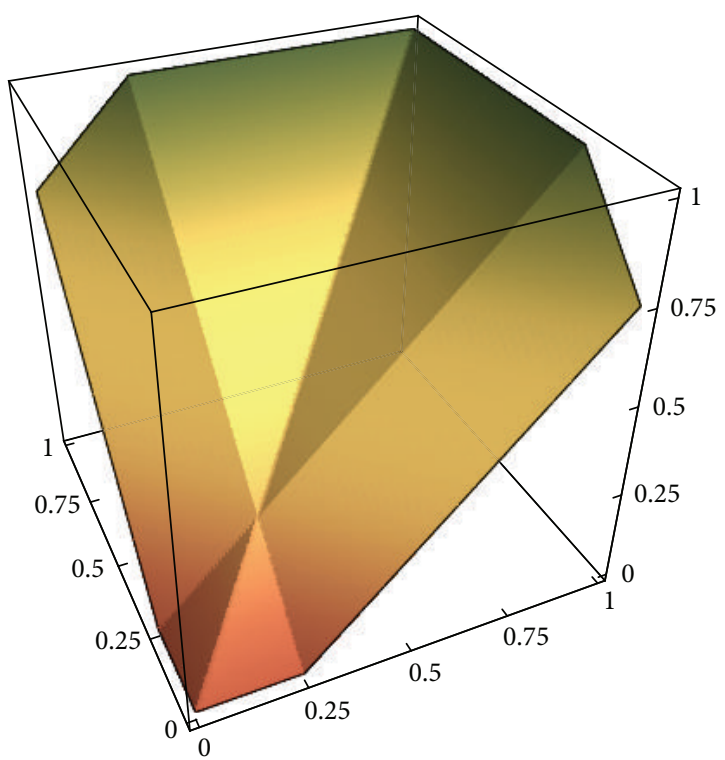

FIGURE 7: The uninorm $U_{T_{M}}$ for $n=4$.

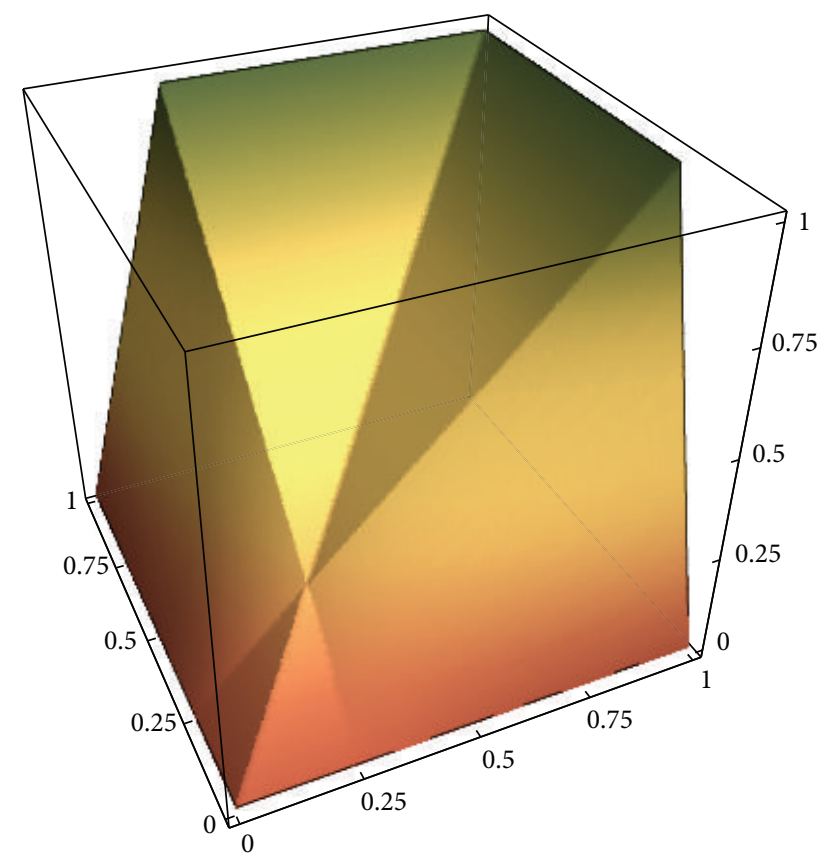

Figure 8: The uninorm $U_{P}$ for $n=4$.

nonnegative vectors given that we are actually considering the asymmetric Choquet integral with respect to $\mu$ (on this, see again Grabisch et al. [20, page 182]).

Definition 6. Let $\mu$ be a capacity on $N$. The Choquet integral with respect to $\mu$ is the function $\mathscr{C}_{\mu}: \mathbb{R}^{n} \rightarrow \mathbb{R}$ given by

$$
\mathscr{C}_{\mu}(\mathbf{x})=\sum_{i=1}^{n} \mu\left(B_{(i)}\right)\left(x_{(i)}-x_{(i-1)}\right),
$$

where $B_{(i)}=\{(i), \ldots,(n)\}$, and one uses the convention $x_{(0)}=$ 0 .

It is worth noting that the Choquet integral has several properties which are useful in certain information aggregation contexts (see, for instance, Grabisch et al. [20, pages 192193 and page 196]).

Remark 7. Let $\mu$ be a capacity on $N$. Then, $\mathscr{C}_{\mu}$ is continuous, monotonic, and homogeneous of degree 1 . Moreover, it is idempotent and compensative when $\mu$ is a normalized capacity.

Notice that the Choquet integral can also be represented by using decreasing sequences of values (see, for instance, Torra [21] and Llamazares [9]):

$$
\mathscr{C}_{\mu}(\mathbf{x})=\sum_{i=1}^{n} \mu\left(A_{[i]}\right)\left(x_{[i]}-x_{[i+1]}\right),
$$

where $A_{[i]}=\{[1], \ldots,[i]\}$, and we use the convention $x_{[n+1]}=$ 0 .

From the previous expression, it is straightforward to show explicitly the weights of the values $x_{[i]}$ by representing the Choquet integral as follows:

$$
\mathscr{C}_{\mu}(\mathbf{x})=\sum_{i=1}^{n}\left(\mu\left(A_{[i]}\right)-\mu\left(A_{[i-1]}\right)\right) x_{[i]} \text {, }
$$

where we use the convention $A_{[0]}=\varnothing$.

3.1. Weighted Means and OWA Operators. Weighted means and OWA operators (Yager [1]) are well-known functions in the field of aggregation operators. Both families of functions are defined in terms of weight distributions that add up to 1.

Definition 8. A vector $\mathbf{q} \in \mathbb{R}^{n}$ is a weighting vector if $\mathbf{q} \in$ $[0,1]^{n}$ and $\sum_{i=1}^{n} q_{i}=1$. $\mathscr{W}_{n}$.

The set of all weighting vectors of $\mathbb{R}^{n}$ will be denoted by

Definition 9. Let $\mathbf{p}$ be a weighting vector. The weighted mean associated with $\mathbf{p}$ is the function $M_{\mathbf{p}}: \mathbb{R}^{n} \rightarrow \mathbb{R}$ given by

$$
M_{\mathbf{p}}(\mathbf{x})=\sum_{i=1}^{n} p_{i} x_{i}
$$

Definition 10. Let $\mathbf{w}$ be a weighting vector. The OWA operator associated with $\mathbf{w}$ is the function $O_{\mathbf{w}}: \mathbb{R}^{n} \rightarrow \mathbb{R}$ given by

$$
\mathrm{O}_{\mathbf{w}}(\mathbf{x})=\sum_{i=1}^{n} w_{i} x_{[i]}
$$

It is well known that weighted means and OWA operators are a special type of Choquet integral (see, for instance, Fodor et al. [22], Grabisch [23, 24], or Llamazares [9]). 
Remark 11. (1) If $\mathbf{p}$ is a weighting vector, then the weighted mean $M_{\mathbf{p}}$ is the Choquet integral with respect to the normalized capacity $\mu_{\mathbf{p}}(A)=\sum_{i \in A} p_{i}$.

(2) If $w$ is a weighting vector, then the OWA operator $O_{w}$ is the Choquet integral with respect to the normalized capacity $\mu_{|\mathbf{w}|}(A)=\sum_{i=1}^{|A|} w_{i}$.

So, according to Remark 7, weighted means and OWA operators are continuous, monotonic, idempotent, compensative, and homogeneous of degree 1. Moreover, in the case of OWA operators, given that the values of the variables are previously ordered in a decreasing way, they are also symmetric.

3.2. SUOWA Operators. SUOWA operators were introduced by Llamazares [9] in order to consider situations where both the importance of information sources and the importance of values had to be taken into account. These functions are Choquet integral-based operators where their capacities are the monotonic cover of certain games. These games are defined by using semiuninorms with neutral element $1 / n$ and the values of the capacities associated with the weighted means and the OWA operators. To be specific, the games from which SUOWA operators are built are defined as follows.

Definition 12. Let $\mathbf{p}$ and $\mathbf{w}$ be two weighting vectors and let $U \in \widetilde{\mathscr{U}}^{1 / n}$.

(1) The game associated with $\mathbf{p}, \mathbf{w}$, and $U$ is the set function $v_{\mathbf{p}, \mathbf{w}}^{U}: 2^{N} \rightarrow \mathbb{R}$ defined by

$$
\begin{aligned}
v_{\mathbf{p}, \mathbf{w}}^{U}(A) & =|A| U\left(\frac{\mu_{\mathbf{p}}(A)}{|A|}, \frac{\mu_{|\mathbf{w}|}(A)}{|A|}\right) \\
& =|A| U\left(\frac{\sum_{i \in A} p_{i}}{|A|}, \frac{\sum_{i=1}^{|A|} w_{i}}{|A|}\right)
\end{aligned}
$$

if $A \neq \varnothing$ and $v_{\mathbf{p}, \mathbf{w}}^{U}(\varnothing)=0$.

(2) $\widehat{v}_{\mathbf{p}, \mathbf{w}}^{U}$, the monotonic cover of the game $v_{\mathbf{p}, \mathbf{w}}^{U}$, will be called the capacity associated with $\mathbf{p}, \mathbf{w}$, and $U$.

Notice that $v_{\mathbf{p}, \mathbf{w}}^{U}(N)=1$. Moreover, since $U \in \widetilde{\mathscr{U}}^{1 / n}$, we have $v_{\mathbf{p}, \mathbf{w}}^{U}(A) \leq 1$ for all $A \subseteq N$ (see Llamazares [9]). Therefore, according to the third item of Remark $5, \widehat{v}_{\mathbf{p}, \mathbf{w}}^{U}$ is always a normalized capacity.

Definition 13. Let $\mathbf{p}$ and $\mathbf{w}$ be two weighting vectors and let $U \in \widetilde{\mathscr{U}}^{1 / n}$. The SUOWA operator associated with $\mathbf{p}, \mathbf{w}$, and $U$ is the function $S_{\mathbf{p}, \mathbf{w}}^{U}: \mathbb{R}^{n} \rightarrow \mathbb{R}$ given by

$$
S_{\mathbf{p}, \mathbf{w}}^{U}(\mathbf{x})=\sum_{i=1}^{n} s_{i} x_{[i]},
$$

where $s_{i}=\widehat{v}_{\mathbf{p}, \mathbf{w}}^{U}\left(A_{[i]}\right)-\widehat{v}_{\mathbf{p}, \mathbf{w}}^{U}\left(A_{[i-1]}\right)$ for all $i \in N, \widehat{v}_{\mathbf{p}, \mathbf{w}}^{U}$ is the capacity associated with $\mathbf{p}, \mathbf{w}$, and $U$, and $A_{[i]}=\{[1], \ldots,[i]\}$ (with the convention that $A_{[0]}=\varnothing$ ).
According to expression (7), the SUOWA operator associated with $\mathbf{p}, \mathbf{w}$, and $U$ can also be written as

$$
S_{\mathbf{p}, \mathbf{w}}^{U}(\mathbf{x})=\sum_{i=1}^{n} \widehat{v}_{\mathbf{p}, \mathbf{w}}^{U}\left(A_{[i]}\right)\left(x_{[i]}-x_{[i+1]}\right) .
$$

By the choice of $\widehat{v}_{\mathbf{p}, \mathbf{w}}^{U}$, we have $S_{\mathbf{p}, \boldsymbol{\eta}}^{U}=M_{\mathbf{p}}$ and $S_{\boldsymbol{\eta}, \mathbf{w}}^{U}=O_{\mathbf{w}}$ for any $U \in \widetilde{\mathscr{U}}^{1 / n}$. Moreover, by Remark 7 and given that $\widehat{v}_{\mathbf{p}, \mathbf{w}}^{U}$ is a normalized capacity, SUOWA operators are continuous, monotonic, idempotent, compensative, and homogeneous of degree 1.

\section{The Results}

The use of Choquet integral has become more and more extensive in the last years (see, for instance, Grabisch et al. [25] and Grabisch and Labreuche [26]). Although simple, the case $n=2$ is interesting from a theoretical point of view. Thus, for instance, Grabisch et al. [20, page 204] show that, in this case, any Choquet integral with respect to a normalized capacity can be written as a convex combination of a minimum, a maximum, and two projections; that is, given a normalized capacity $\mu$, there exists a weighting vector $\lambda$ belonging to $\mathscr{W}_{4}$ such that

$$
\begin{aligned}
\mathscr{C}_{\mu}\left(x_{1}, x_{2}\right)= & \lambda_{1} \min \left(x_{1}, x_{2}\right)+\lambda_{2} \max \left(x_{1}, x_{2}\right) \\
& +\lambda_{3} x_{1}+\lambda_{4} x_{2} .
\end{aligned}
$$

In our case, we are going to show that any Choquet integral with respect to a normalized capacity can be written as a SUOWA operator. Notice that when $n=2, v_{\mathbf{p}, \mathbf{w}}^{U}$ is always a normalized capacity for any weighting vectors $\mathbf{p}$ and $\mathbf{w}$ and for any semiuninorm $U$. Therefore, given a normalized capacity $\mu$, we need to prove that there exist weighting vectors $\mathbf{p}$ and $\mathbf{w}$ and a semiuninorm $U$ such that

$$
\begin{aligned}
& v_{\mathbf{p}, \mathbf{w}}^{U}(\{1\})=U\left(p_{1}, w_{1}\right)=\mu_{1}, \\
& v_{\mathbf{p}, \mathbf{w}}^{U}(\{2\})=U\left(p_{2}, w_{1}\right)=\mu_{2},
\end{aligned}
$$

where we use the notations $\mu_{1}$ and $\mu_{2}$ to denote the values $\mu(\{1\})$ and $\mu(\{2\})$, respectively.

Firstly we are going to show that, in the case of the semiuninorms $U_{\perp}, U_{\mathrm{T}}, U_{\min }$, and $U_{\text {max }}$, there exist normalized capacities which cannot be expressed as SUOWA operators. For this, we will use the following lemma.

Lemma 14. If $U \in\left\{U_{\perp}, U_{\mathrm{T}}, U_{\min }, U_{\max }\right\}$, then $U(x, y)=0.5$ if and only if $x=y=0.5$.

Proof. Let $U \in\left\{U_{\perp}, U_{\mathrm{T}}, U_{\min }, U_{\max }\right\}$. Since 0.5 is the neutral element of $U$, we have $U(0.5,0.5)=0.5$.

Conversely, suppose $U(x, y)=0.5$. In Table 1 , where $0.5^{-}$ stands for a value that belongs to $[0,0.5)$ and $0.5^{+}$stands for a value that belongs to $(0.5,1]$, we show the values taken by the semiuninorms $U_{\perp}, U_{\min }, U_{\max }$, and $U_{\mathrm{T}}$ when $(x, y) \in[0,1]^{2}$. Therefore, if $U(x, y)=0.5$, then necessarily $x=y=0.5$. 
TABLE 1: Values taken by $U_{\perp}, U_{\min }, U_{\max }$, and $U_{\mathrm{T}}$.

\begin{tabular}{llcccc}
\hline$x$ & $y$ & $U_{\perp}(x, y)$ & $U_{\min }(x, y)$ & $U_{\max }(x, y)$ & $U_{\mathrm{T}}(x, y)$ \\
\hline $0.5^{-}$ & $0.5^{-}$ & 0 & $\min (x, y)$ & $\min (x, y)$ & $\min (x, y)$ \\
$0.5^{-}$ & 0.5 & $x$ & $x$ & $x$ & $x$ \\
$0.5^{-}$ & $0.5^{+}$ & $x$ & $x$ & $y$ & $y$ \\
0.5 & $0.5^{-}$ & $y$ & $y$ & $y$ & $y$ \\
0.5 & 0.5 & 0.5 & 0.5 & 0.5 & 0.5 \\
0.5 & $0.5^{+}$ & $y$ & $y$ & $y$ & $y$ \\
$0.5^{+}$ & $0.5^{-}$ & $y$ & $y$ & $x$ & $x$ \\
$0.5^{+}$ & 0.5 & $x$ & $x$ & $x$ & $x$ \\
$0.5^{+}$ & $0.5^{+}$ & $\max (x, y)$ & $\max (x, y)$ & $\max (x, y)$ & 1 \\
\hline
\end{tabular}

Theorem 15. Let $\mu$ be the normalized capacity on $N=\{1,2\}$ such that $\mu_{1}=0$ and $\mu_{2}=0.5$. If $U \in\left\{U_{\perp}, U_{\mathrm{T}}, U_{\min }, U_{\max }\right\}$, then there do not exist weighting vectors $\mathbf{p}$ and $\mathbf{w}$ such that $\mu=$ $v_{\mathbf{p}, \mathbf{w}}^{U}$.

Proof. Given $U \in\left\{U_{\perp}, U_{\mathrm{T}}, U_{\min }, U_{\max }\right\}$, consider two weighting vectors $\mathbf{p}$ and $\mathbf{w}$ such that $U\left(p_{2}, w_{1}\right)=0.5$. By Lemma 14 , we have $p_{2}=w_{1}=0.5$. Therefore, $U\left(p_{1}, w_{1}\right)=U(0.5,0.5)=$ 0.5 and, consequently, $U\left(p_{1}, w_{1}\right)=0$ is not possible.

In each of the following theorems we consider the semiuninorms $U_{T_{\mathrm{L}}}, U_{T_{\mathrm{M}}}, U_{\widetilde{P}}$, and $U_{P}$, respectively, and we show that any normalized capacity can be written as a SUOWA operator associated with appropriate weighting vectors $\mathbf{p}$ and $\mathbf{w}$, which are given explicitly.

Theorem 16. Let $\mu$ be a normalized capacity on $N=\{1,2\}$ and let $\mathbf{p}$ and $\mathbf{w}$ be two weighting vectors defined as follows:

(1) If $\mu_{1}+\mu_{2}<1$, then

$$
\begin{aligned}
& \mathbf{p}=\left(0.5+\frac{\mu_{1}-\mu_{2}}{2}, 0.5+\frac{\mu_{2}-\mu_{1}}{2}\right), \\
& \mathbf{w}=\left(\frac{\mu_{1}+\mu_{2}}{2}, 1-\frac{\mu_{1}+\mu_{2}}{2}\right) .
\end{aligned}
$$

(2) If $\mu_{1}+\mu_{2} \geq 1$ and $\min \left(\mu_{1}, \mu_{2}\right)<0.5$, then

$$
\begin{aligned}
& \mathbf{p}= \begin{cases}\left(\mu_{1}, 1-\mu_{1}\right) & \text { if } \mu_{1}>0.5, \\
\left(1-\mu_{2}, \mu_{2}\right) & \text { if } \mu_{2}>0.5,\end{cases} \\
& \mathbf{w}=\left(\mu_{1}+\mu_{2}-0.5,1.5-\mu_{1}-\mu_{2}\right) .
\end{aligned}
$$

(3) If $\min \left(\mu_{1}, \mu_{2}\right) \geq 0.5$, then

$$
\begin{aligned}
& \mathbf{p}=\left(0.5+\mu_{1}-\mu_{2}, 0.5+\mu_{2}-\mu_{1}\right), \\
& \mathbf{w}=\left(\max \left(\mu_{1}, \mu_{2}\right), 1-\max \left(\mu_{1}, \mu_{2}\right)\right) .
\end{aligned}
$$

Then, $\mu=v_{\mathbf{p}, \mathbf{w}}^{U_{T_{\mathbf{L}}}}$; that is, $\mathscr{C}_{\mu}=S_{\mathbf{p}, \mathbf{w}}^{U_{T_{\mathbf{L}}}}$.
Proof. Let $\mu$ be a normalized capacity on $N=\{1,2\}$ and recall that when $n=2$, the semiuninorm $U_{T_{\mathbf{L}}}$ is defined by

$$
\begin{aligned}
& U_{T_{\mathrm{L}}}(x, y) \\
& \quad= \begin{cases}\max (x, y) & \text { if }(x, y) \in[0.5,1]^{2}, \\
\max (x+y-0.5,0) & \text { otherwise. }\end{cases}
\end{aligned}
$$

We distinguish the following cases:

(1) If $\mu_{1}+\mu_{2}<1$, consider

$$
\begin{aligned}
& \mathbf{p}=\left(0.5+\frac{\mu_{1}-\mu_{2}}{2}, 0.5+\frac{\mu_{2}-\mu_{1}}{2}\right), \\
& \mathbf{w}=\left(\frac{\mu_{1}+\mu_{2}}{2}, 1-\frac{\mu_{1}+\mu_{2}}{2}\right) .
\end{aligned}
$$

Then,

$$
\begin{aligned}
& U_{T_{\mathrm{L}}}\left(p_{1}, w_{1}\right)=0.5+\frac{\mu_{1}-\mu_{2}}{2}+\frac{\mu_{1}+\mu_{2}}{2}-0.5=\mu_{1}, \\
& U_{T_{\mathrm{L}}}\left(p_{2}, w_{1}\right)=0.5+\frac{\mu_{2}-\mu_{1}}{2}+\frac{\mu_{1}+\mu_{2}}{2}-0.5=\mu_{2} .
\end{aligned}
$$

(2) If $\mu_{1}+\mu_{2} \geq 1$ and $\min \left(\mu_{1}, \mu_{2}\right)<0.5$, consider

$$
\begin{gathered}
\mathbf{p}= \begin{cases}\left(\mu_{1}, 1-\mu_{1}\right) & \text { if } \mu_{1}>0.5, \\
\left(1-\mu_{2}, \mu_{2}\right) & \text { if } \mu_{2}>0.5,\end{cases} \\
\mathbf{w}=\left(\mu_{1}+\mu_{2}-0.5,1.5-\mu_{1}-\mu_{2}\right) .
\end{gathered}
$$

We distinguish two cases:

(a) If $\mu_{1}>0.5$, then

$$
\begin{aligned}
& U_{T_{\mathbf{L}}}\left(p_{1}, w_{1}\right)=\max \left(\mu_{1}, \mu_{1}+\mu_{2}-0.5\right)=\mu_{1}, \\
& U_{T_{\mathbf{L}}}\left(p_{2}, w_{1}\right)=1-\mu_{1}+\mu_{1}+\mu_{2}-0.5-0.5=\mu_{2} .
\end{aligned}
$$

(b) If $\mu_{2}>0.5$, then

$$
\begin{aligned}
& U_{T_{\mathbf{L}}}\left(p_{1}, w_{1}\right)=1-\mu_{2}+\mu_{1}+\mu_{2}-0.5-0.5=\mu_{1}, \\
& U_{T_{\mathbf{L}}}\left(p_{2}, w_{1}\right)=\max \left(\mu_{2}, \mu_{1}+\mu_{2}-0.5\right)=\mu_{2} .
\end{aligned}
$$

(3) If $\min \left(\mu_{1}, \mu_{2}\right) \geq 0.5$, consider

$$
\begin{aligned}
& \mathbf{p}=\left(0.5+\mu_{1}-\mu_{2}, 0.5+\mu_{2}-\mu_{1}\right), \\
& \mathbf{w}=\left(\max \left(\mu_{1}, \mu_{2}\right), 1-\max \left(\mu_{1}, \mu_{2}\right)\right) .
\end{aligned}
$$

We distinguish three cases:

(a) If $\mu_{1}>\mu_{2}$, then

$$
\begin{aligned}
& U_{T_{\mathbf{L}}}\left(p_{1}, w_{1}\right)=\max \left(0.5+\mu_{1}-\mu_{2}, \mu_{1}\right)=\mu_{1}, \\
& U_{T_{\mathbf{L}}}\left(p_{2}, w_{1}\right)=0.5+\mu_{2}-\mu_{1}+\mu_{1}-0.5=\mu_{2} .
\end{aligned}
$$


(b) If $\mu_{1}=\mu_{2}$, then

$$
\begin{aligned}
& U_{T_{\mathbf{L}}}\left(p_{1}, w_{1}\right)=\max \left(0.5, \mu_{1}\right)=\mu_{1}, \\
& U_{T_{\mathbf{L}}}\left(p_{2}, w_{1}\right)=\max \left(0.5, \mu_{2}\right)=\mu_{2} .
\end{aligned}
$$

(c) If $\mu_{1}<\mu_{2}$, then

$$
\begin{aligned}
& U_{T_{\mathbf{L}}}\left(p_{1}, w_{1}\right)=0.5+\mu_{1}-\mu_{2}+\mu_{2}-0.5=\mu_{1}, \\
& U_{T_{\mathbf{L}}}\left(p_{2}, w_{1}\right)=\max \left(0.5+\mu_{2}-\mu_{1}, \mu_{2}\right)=\mu_{2} .
\end{aligned}
$$

Theorem 17. Let $\mu$ be a normalized capacity on $N=\{1,2\}$ and let $\mathbf{p}$ and $\mathbf{w}$ be two weighting vectors defined as follows:

(1) If $\max \left(\mu_{1}, \mu_{2}\right)<0.5$, then

$$
\begin{aligned}
& \mathbf{p}=\left(0.5+\mu_{1}-\mu_{2}, 0.5+\mu_{2}-\mu_{1}\right), \\
& \mathbf{w}=\left(\min \left(\mu_{1}, \mu_{2}\right), 1-\min \left(\mu_{1}, \mu_{2}\right)\right) .
\end{aligned}
$$

(2) If $\mu_{1}+\mu_{2}<1$ and $\max \left(\mu_{1}, \mu_{2}\right) \geq 0.5$, then

$$
\begin{aligned}
& \mathbf{p}= \begin{cases}\left(\mu_{1}, 1-\mu_{1}\right) & \text { if } \mu_{1}<0.5, \\
\left(1-\mu_{2}, \mu_{2}\right) & \text { if } \mu_{2}<0.5,\end{cases} \\
& \mathbf{w}=\left(\mu_{1}+\mu_{2}-0.5,1.5-\mu_{1}-\mu_{2}\right) \text {. }
\end{aligned}
$$

(3) If $\mu_{1}+\mu_{2} \geq 1$ and $\min \left(\mu_{1}, \mu_{2}\right)<0.5$, then

$$
\begin{aligned}
& \mathbf{p}= \begin{cases}\left(\mu_{1}, 1-\mu_{1}\right) & \text { if } \mu_{1}>0.5, \\
\left(1-\mu_{2}, \mu_{2}\right) & \text { if } \mu_{2}>0.5,\end{cases} \\
& \mathbf{w}=\left(\mu_{1}+\mu_{2}-0.5,1.5-\mu_{1}-\mu_{2}\right) \text {. }
\end{aligned}
$$

(4) If $\min \left(\mu_{1}, \mu_{2}\right) \geq 0.5$, then

$$
\begin{aligned}
\mathbf{p} & =\left(0.5+\mu_{1}-\mu_{2}, 0.5+\mu_{2}-\mu_{1}\right), \\
\mathbf{w} & =\left(\max \left(\mu_{1}, \mu_{2}\right), 1-\max \left(\mu_{1}, \mu_{2}\right)\right) .
\end{aligned}
$$

Then, $\mu=v_{\mathbf{p}, \mathbf{w}}^{U_{T_{M}}}$; that is, $\mathscr{C}_{\mu}=S_{\mathbf{p}, \mathbf{w}}^{U_{T_{\mathbf{w}}}}$.

Proof. Let $\mu$ be a normalized capacity on $N=\{1,2\}$ and recall that when $n=2$, the semiuninorm $U_{T_{M}}$ is defined by

$$
U_{T_{M}}(x, y)= \begin{cases}\max (x, y) & \text { if }(x, y) \in[0.5,1]^{2}, \\ \min (x, y) & \text { if }(x, y) \in[0,0.5)^{2}, \\ x+y-0.5 & \text { otherwise. }\end{cases}
$$

We distinguish the following cases:

(1) If $\max \left(\mu_{1}, \mu_{2}\right)<0.5$, consider

$$
\begin{aligned}
& \mathbf{p}=\left(0.5+\mu_{1}-\mu_{2}, 0.5+\mu_{2}-\mu_{1}\right), \\
& \mathbf{w}=\left(\min \left(\mu_{1}, \mu_{2}\right), 1-\min \left(\mu_{1}, \mu_{2}\right)\right) .
\end{aligned}
$$

We distinguish three cases:

(a) If $\mu_{1}<\mu_{2}$, then

$$
\begin{aligned}
& U_{T_{M}}\left(p_{1}, w_{1}\right)=\min \left(0.5+\mu_{1}-\mu_{2}, \mu_{1}\right)=\mu_{1}, \\
& U_{T_{M}}\left(p_{2}, w_{1}\right)=0.5+\mu_{2}-\mu_{1}+\mu_{1}-0.5=\mu_{2} .
\end{aligned}
$$

(b) If $\mu_{1}=\mu_{2}$, then

$$
\begin{aligned}
& U_{T_{M}}\left(p_{1}, w_{1}\right)=\min \left(0.5, \mu_{1}\right)=\mu_{1}, \\
& U_{T_{M}}\left(p_{2}, w_{1}\right)=\min \left(0.5, \mu_{2}\right)=\mu_{2} .
\end{aligned}
$$

(c) If $\mu_{1}>\mu_{2}$, then

$$
\begin{aligned}
& U_{T_{M}}\left(p_{1}, w_{1}\right)=0.5+\mu_{1}-\mu_{2}+\mu_{2}-0.5=\mu_{1}, \\
& U_{T_{M}}\left(p_{2}, w_{1}\right)=\min \left(0.5+\mu_{2}-\mu_{1}, \mu_{2}\right)=\mu_{2} .
\end{aligned}
$$

(2) If $\mu_{1}+\mu_{2}<1$ and $\max \left(\mu_{1}, \mu_{2}\right) \geq 0.5$, consider

$$
\begin{aligned}
& \mathbf{p}= \begin{cases}\left(\mu_{1}, 1-\mu_{1}\right) & \text { if } \mu_{1}<0.5, \\
\left(1-\mu_{2}, \mu_{2}\right) & \text { if } \mu_{2}<0.5,\end{cases} \\
& \mathbf{w}=\left(\mu_{1}+\mu_{2}-0.5,1.5-\mu_{1}-\mu_{2}\right) .
\end{aligned}
$$

We distinguish two cases:

(a) If $\mu_{1}<0.5$, then

$$
\begin{aligned}
& U_{T_{M}}\left(p_{1}, w_{1}\right)=\min \left(\mu_{1}, \mu_{1}+\mu_{2}-0.5\right)=\mu_{1}, \\
& U_{T_{M}}\left(p_{2}, w_{1}\right)=1-\mu_{1}+\mu_{1}+\mu_{2}-0.5-0.5=\mu_{2} .
\end{aligned}
$$

(b) If $\mu_{2}<0.5$, then

$$
\begin{aligned}
& U_{T_{M}}\left(p_{1}, w_{1}\right)=1-\mu_{2}+\mu_{1}+\mu_{2}-0.5-0.5=\mu_{1}, \\
& U_{T_{M}}\left(p_{2}, w_{1}\right)=\min \left(\mu_{2}, \mu_{1}+\mu_{2}-0.5\right)=\mu_{2} .
\end{aligned}
$$

(3) If $\mu_{1}+\mu_{2} \geq 1$ and $\min \left(\mu_{1}, \mu_{2}\right)<0.5$, then the proof of this case is similar to that of the second item in Theorem 16.

(4) If $\min \left(\mu_{1}, \mu_{2}\right) \geq 0.5$, then the proof of this case is similar to that of the third item in Theorem 16.

Theorem 18. Let $\mu$ be a normalized capacity on $N=\{1,2\}$ and let $\mathbf{p}$ and $\mathbf{w}$ be two weighting vectors defined as follows:

(1) If $\mu_{1}+\mu_{2}<1$, then

$$
\begin{aligned}
& \mathbf{p}= \begin{cases}\left(p_{1}, p_{2}\right) \in \mathscr{W}_{2} & \text { if } \mu_{1}=\mu_{2}=0, \\
\left(\frac{\mu_{1}}{\mu_{1}+\mu_{2}}, \frac{\mu_{2}}{\mu_{1}+\mu_{2}}\right) & \text { otherwise, }\end{cases} \\
& \mathbf{w}=\left(\frac{\mu_{1}+\mu_{2}}{2}, 1-\frac{\mu_{1}+\mu_{2}}{2}\right) .
\end{aligned}
$$


(2) If $\mu_{1}+\mu_{2} \geq 1$ and $\min \left(\mu_{1}, \mu_{2}\right)<2 \max \left(\mu_{1}, \mu_{2}\right)(1-$ $\left.\max \left(\mu_{1}, \mu_{2}\right)\right)$, then

$$
\begin{aligned}
\mathbf{p} & = \begin{cases}\left(\mu_{1}, 1-\mu_{1}\right) & \text { if } \mu_{1} \geq \mu_{2}, \\
\left(1-\mu_{2}, \mu_{2}\right) & \text { if } \mu_{1}<\mu_{2},\end{cases} \\
\mathbf{w} & =\left(\frac{\min \left(\mu_{1}, \mu_{2}\right)}{2\left(1-\max \left(\mu_{1}, \mu_{2}\right)\right)}, 1\right. \\
& \left.-\frac{\min \left(\mu_{1}, \mu_{2}\right)}{2\left(1-\max \left(\mu_{1}, \mu_{2}\right)\right)}\right) .
\end{aligned}
$$

(3) If $\mu_{1}+\mu_{2} \geq 1$ and $\min \left(\mu_{1}, \mu_{2}\right) \geq 2 \max \left(\mu_{1}, \mu_{2}\right)(1-$ $\left.\max \left(\mu_{1}, \mu_{2}\right)\right)$, then

$$
\begin{aligned}
& \mathbf{p}= \begin{cases}\left(1-\frac{\mu_{2}}{2 \mu_{1}}, \frac{\mu_{2}}{2 \mu_{1}}\right) & \text { if } \mu_{1} \geq \mu_{2}, \\
\left(\frac{\mu_{1}}{2 \mu_{2}}, 1-\frac{\mu_{1}}{2 \mu_{2}}\right) & \text { if } \mu_{1}<\mu_{2},\end{cases} \\
& \mathbf{w}=\left(\max \left(\mu_{1}, \mu_{2}\right), 1-\max \left(\mu_{1}, \mu_{2}\right)\right) .
\end{aligned}
$$

Then, $\mu=v_{\mathbf{p}, \mathbf{w}}^{U_{\tilde{P}}}$; that is, $\mathscr{C}_{\mu}=S_{\mathbf{p}, \mathbf{w}}^{U_{\tilde{T}}}$.

Proof. Let $\mu$ be a normalized capacity on $N=\{1,2\}$ and recall that when $n=2$, the semiuninorm $U_{\widetilde{P}}$ is defined by

$$
U_{\widetilde{P}}(x, y)= \begin{cases}\max (x, y) & \text { if }(x, y) \in[0.5,1]^{2}, \\ 2 x y & \text { otherwise. }\end{cases}
$$

We distinguish the following cases:

(1) If $\mu_{1}+\mu_{2}<1$, consider

$$
\begin{aligned}
& \mathbf{p}= \begin{cases}\left(p_{1}, p_{2}\right) \in \mathscr{W}_{2} & \text { if } \mu_{1}=\mu_{2}=0, \\
\left(\frac{\mu_{1}}{\mu_{1}+\mu_{2}}, \frac{\mu_{2}}{\mu_{1}+\mu_{2}}\right) & \text { otherwise, }\end{cases} \\
& \mathbf{w}=\left(\frac{\mu_{1}+\mu_{2}}{2}, 1-\frac{\mu_{1}+\mu_{2}}{2}\right) .
\end{aligned}
$$

We distinguish two cases:

(a) If $\mu_{1}=\mu_{2}=0$, then

$$
\begin{aligned}
& U_{\widetilde{P}}\left(p_{1}, w_{1}\right)=2 \cdot p_{1} \cdot 0=0=\mu_{1}, \\
& U_{\widetilde{P}}\left(p_{2}, w_{1}\right)=2 \cdot p_{2} \cdot 0=0=\mu_{2} .
\end{aligned}
$$

(b) If $\left(\mu_{1}, \mu_{2}\right) \neq(0,0)$, then

$$
\begin{aligned}
& U_{\widetilde{P}}\left(p_{1}, w_{1}\right)=2 \frac{\mu_{1}}{\mu_{1}+\mu_{2}} \frac{\mu_{1}+\mu_{2}}{2}=\mu_{1}, \\
& U_{\widetilde{P}}\left(p_{2}, w_{1}\right)=2 \frac{\mu_{2}}{\mu_{1}+\mu_{2}} \frac{\mu_{1}+\mu_{2}}{2}=\mu_{2} .
\end{aligned}
$$

(2) If $\mu_{1}+\mu_{2} \geq 1$ and $\min \left(\mu_{1}, \mu_{2}\right)<2 \max \left(\mu_{1}, \mu_{2}\right)(1-$ $\left.\max \left(\mu_{1}, \mu_{2}\right)\right)$, then notice that the case $\max \left(\mu_{1}, \mu_{2}\right)=1$ is not possible. Moreover, we have

$$
\frac{\min \left(\mu_{1}, \mu_{2}\right)}{2\left(1-\max \left(\mu_{1}, \mu_{2}\right)\right)}<\max \left(\mu_{1}, \mu_{2}\right) .
$$

On the other hand, given that $\min \left(\mu_{1}, \mu_{2}\right) \geq 1-\max \left(\mu_{1}, \mu_{2}\right)$, we get

$$
\frac{\min \left(\mu_{1}, \mu_{2}\right)}{2\left(1-\max \left(\mu_{1}, \mu_{2}\right)\right)} \geq 0.5,
$$

and, consequently, $\max \left(\mu_{1}, \mu_{2}\right)>0.5$. Now consider the following weighting vectors:

$$
\begin{aligned}
& \mathbf{p}= \begin{cases}\left(\mu_{1}, 1-\mu_{1}\right) & \text { if } \mu_{1} \geq \mu_{2}, \\
\left(1-\mu_{2}, \mu_{2}\right) & \text { if } \mu_{1}<\mu_{2},\end{cases} \\
& \mathbf{w} \\
& =\left(\frac{\min \left(\mu_{1}, \mu_{2}\right)}{2\left(1-\max \left(\mu_{1}, \mu_{2}\right)\right)}, 1-\frac{\min \left(\mu_{1}, \mu_{2}\right)}{2\left(1-\max \left(\mu_{1}, \mu_{2}\right)\right)}\right) .
\end{aligned}
$$

We distinguish two cases:

(a) If $\mu_{1} \geq \mu_{2}$, then

$$
\begin{aligned}
& U_{\widetilde{P}}\left(p_{1}, w_{1}\right)=\max \left(\mu_{1}, \frac{\mu_{2}}{2\left(1-\mu_{1}\right)}\right)=\mu_{1}, \\
& U_{\widetilde{P}}\left(p_{2}, w_{1}\right)=2\left(1-\mu_{1}\right) \frac{\mu_{2}}{2\left(1-\mu_{1}\right)}=\mu_{2} .
\end{aligned}
$$

(b) If $\mu_{1}<\mu_{2}$, then

$$
\begin{aligned}
& U_{\widetilde{P}}\left(p_{1}, w_{1}\right)=2\left(1-\mu_{2}\right) \frac{\mu_{1}}{2\left(1-\mu_{2}\right)}=\mu_{1}, \\
& U_{\widetilde{P}}\left(p_{2}, w_{1}\right)=\max \left(\mu_{2}, \frac{\mu_{1}}{2\left(1-\mu_{2}\right)}\right)=\mu_{2} .
\end{aligned}
$$

(3) If $\mu_{1}+\mu_{2} \geq 1$ and $\min \left(\mu_{1}, \mu_{2}\right) \geq 2 \max \left(\mu_{1}, \mu_{2}\right)(1-$ $\left.\max \left(\mu_{1}, \mu_{2}\right)\right)$, then $\max \left(\mu_{1}, \mu_{2}\right) \geq 0.5$, and we also have

$$
\frac{\min \left(\mu_{1}, \mu_{2}\right)}{2 \max \left(\mu_{1}, \mu_{2}\right)} \geq 1-\max \left(\mu_{1}, \mu_{2}\right),
$$

or, equivalently,

$$
1-\frac{\min \left(\mu_{1}, \mu_{2}\right)}{2 \max \left(\mu_{1}, \mu_{2}\right)} \leq \max \left(\mu_{1}, \mu_{2}\right) .
$$

On the other hand, since $\min \left(\mu_{1}, \mu_{2}\right) \leq \max \left(\mu_{1}, \mu_{2}\right)$, we get

$$
\frac{\min \left(\mu_{1}, \mu_{2}\right)}{2 \max \left(\mu_{1}, \mu_{2}\right)} \leq 0.5,
$$


and, consequently,

$$
1-\frac{\min \left(\mu_{1}, \mu_{2}\right)}{2 \max \left(\mu_{1}, \mu_{2}\right)} \geq 0.5 .
$$

Consider now the following weighting vectors:

$$
\begin{aligned}
& \mathbf{p}= \begin{cases}\left(1-\frac{\mu_{2}}{2 \mu_{1}}, \frac{\mu_{2}}{2 \mu_{1}}\right) & \text { if } \mu_{1} \geq \mu_{2}, \\
\left(\frac{\mu_{1}}{2 \mu_{2}}, 1-\frac{\mu_{1}}{2 \mu_{2}}\right) & \text { if } \mu_{1}<\mu_{2},\end{cases} \\
& \mathbf{w}=\left(\max \left(\mu_{1}, \mu_{2}\right), 1-\max \left(\mu_{1}, \mu_{2}\right)\right) .
\end{aligned}
$$

We distinguish three cases:

(a) If $\mu_{1}>\mu_{2}$, then

$$
\begin{aligned}
& U_{\widetilde{P}}\left(p_{1}, w_{1}\right)=\max \left(1-\frac{\mu_{2}}{2 \mu_{1}}, \mu_{1}\right)=\mu_{1}, \\
& U_{\widetilde{P}}\left(p_{2}, w_{1}\right)=2 \frac{\mu_{2}}{2 \mu_{1}} \mu_{1}=\mu_{2} .
\end{aligned}
$$

(b) If $\mu_{1}=\mu_{2}$, then

$$
\begin{aligned}
& U_{\widetilde{P}}\left(p_{1}, w_{1}\right)=\max \left(0.5, \mu_{1}\right)=\mu_{1}, \\
& U_{\widetilde{P}}\left(p_{2}, w_{1}\right)=\max \left(0.5, \mu_{2}\right)=\mu_{2} .
\end{aligned}
$$

(c) If $\mu_{1}<\mu_{2}$, then

$$
\begin{aligned}
& U_{\widetilde{P}}\left(p_{1}, w_{1}\right)=2 \frac{\mu_{1}}{2 \mu_{2}} \mu_{2}=\mu_{1}, \\
& U_{\widetilde{P}}\left(p_{2}, w_{1}\right)=\max \left(1-\frac{\mu_{1}}{2 \mu_{2}}, \mu_{2}\right)=\mu_{2} .
\end{aligned}
$$

Theorem 19. Let $\mu$ be a normalized capacity on $N=\{1,2\}$ and let $\mathbf{p}$ and $\mathbf{w}$ be two weighting vectors defined as follows:

(1) If $\mu_{1}+\mu_{2}<1$ and $\max \left(\mu_{1}, \mu_{2}\right)<2 \min \left(\mu_{1}, \mu_{2}\right)(1-$ $\left.\min \left(\mu_{1}, \mu_{2}\right)\right)$, then

$$
\begin{aligned}
& \mathbf{p}= \begin{cases}\left(1-\frac{\mu_{2}}{2 \mu_{1}}, \frac{\mu_{2}}{2 \mu_{1}}\right) & \text { if } \mu_{1} \leq \mu_{2}, \\
\left(\frac{\mu_{1}}{2 \mu_{2}}, 1-\frac{\mu_{1}}{2 \mu_{2}}\right) & \text { if } \mu_{1}>\mu_{2},\end{cases} \\
& \mathbf{w}=\left(\min \left(\mu_{1}, \mu_{2}\right), 1-\min \left(\mu_{1}, \mu_{2}\right)\right) .
\end{aligned}
$$

(2) If $\mu_{1}+\mu_{2}<1$ and $\max \left(\mu_{1}, \mu_{2}\right) \geq 2 \min \left(\mu_{1}, \mu_{2}\right)(1-$ $\left.\min \left(\mu_{1}, \mu_{2}\right)\right)$, then

$\mathbf{p}= \begin{cases}\left(\mu_{1}, 1-\mu_{1}\right) & \text { if } \mu_{1} \leq \mu_{2}, \\ \left(1-\mu_{2}, \mu_{2}\right) & \text { if } \mu_{1}>\mu_{2},\end{cases}$

w

$$
=\left(\frac{\max \left(\mu_{1}, \mu_{2}\right)}{2\left(1-\min \left(\mu_{1}, \mu_{2}\right)\right)}, 1-\frac{\max \left(\mu_{1}, \mu_{2}\right)}{2\left(1-\min \left(\mu_{1}, \mu_{2}\right)\right)}\right) .
$$

(3) If $\mu_{1}+\mu_{2} \geq 1$ and $\min \left(\mu_{1}, \mu_{2}\right)<2 \max \left(\mu_{1}, \mu_{2}\right)(1-$ $\left.\max \left(\mu_{1}, \mu_{2}\right)\right)$, then

$\mathbf{p}= \begin{cases}\left(\mu_{1}, 1-\mu_{1}\right) & \text { if } \mu_{1} \geq \mu_{2}, \\ \left(1-\mu_{2}, \mu_{2}\right) & \text { if } \mu_{1}<\mu_{2},\end{cases}$

w

$$
=\left(\frac{\min \left(\mu_{1}, \mu_{2}\right)}{2\left(1-\max \left(\mu_{1}, \mu_{2}\right)\right)}, 1-\frac{\min \left(\mu_{1}, \mu_{2}\right)}{2\left(1-\max \left(\mu_{1}, \mu_{2}\right)\right)}\right) .
$$

(4) If $\mu_{1}+\mu_{2} \geq 1$ and $\min \left(\mu_{1}, \mu_{2}\right) \geq 2 \max \left(\mu_{1}, \mu_{2}\right)(1-$ $\left.\max \left(\mu_{1}, \mu_{2}\right)\right)$, then

$$
\begin{aligned}
& \mathbf{p}= \begin{cases}\left(1-\frac{\mu_{2}}{2 \mu_{1}}, \frac{\mu_{2}}{2 \mu_{1}}\right) & \text { if } \mu_{1} \geq \mu_{2}, \\
\left(\frac{\mu_{1}}{2 \mu_{2}}, 1-\frac{\mu_{1}}{2 \mu_{2}}\right) & \text { if } \mu_{1}<\mu_{2},\end{cases} \\
& \mathbf{w}=\left(\max \left(\mu_{1}, \mu_{2}\right), 1-\max \left(\mu_{1}, \mu_{2}\right)\right) .
\end{aligned}
$$

Then, $\mu=v_{\mathbf{p}, \mathbf{w}}^{U_{P}}$; that is, $\mathscr{C}_{\mu}=S_{\mathbf{p}, \mathbf{w}}^{U_{P}}$.

Proof. Let $\mu$ be a normalized capacity on $N=\{1,2\}$ and recall that when $n=2$, the semiuninorm $U_{P}$ is defined by

$$
U_{P}(x, y)= \begin{cases}\max (x, y) & \text { if }(x, y) \in[0.5,1]^{2}, \\ \min (x, y) & \text { if }(x, y) \in[0,0.5)^{2} \\ 2 x y & \text { otherwise. }\end{cases}
$$

We distinguish the following cases:

(1) If $\mu_{1}+\mu_{2}<1$ and $\max \left(\mu_{1}, \mu_{2}\right)<2 \min \left(\mu_{1}, \mu_{2}\right)(1-$ $\left.\min \left(\mu_{1}, \mu_{2}\right)\right)$, then notice that the case $\min \left(\mu_{1}, \mu_{2}\right)=0$ is not possible. Moreover, $\min \left(\mu_{1}, \mu_{2}\right)<0.5$, and we also have

$$
\frac{\max \left(\mu_{1}, \mu_{2}\right)}{2 \min \left(\mu_{1}, \mu_{2}\right)}<1-\min \left(\mu_{1}, \mu_{2}\right)
$$

or, equivalently,

$$
\min \left(\mu_{1}, \mu_{2}\right)<1-\frac{\max \left(\mu_{1}, \mu_{2}\right)}{2 \min \left(\mu_{1}, \mu_{2}\right)} .
$$

On the other hand, since $\min \left(\mu_{1}, \mu_{2}\right) \leq \max \left(\mu_{1}, \mu_{2}\right)$, we get

$$
\frac{\max \left(\mu_{1}, \mu_{2}\right)}{2 \min \left(\mu_{1}, \mu_{2}\right)} \geq 0.5 \text {, }
$$

and, consequently,

$$
1-\frac{\max \left(\mu_{1}, \mu_{2}\right)}{2 \min \left(\mu_{1}, \mu_{2}\right)} \leq 0.5 .
$$

Consider now the following weighting vectors:

$$
\begin{aligned}
& \mathbf{p}= \begin{cases}\left(1-\frac{\mu_{2}}{2 \mu_{1}}, \frac{\mu_{2}}{2 \mu_{1}}\right) & \text { if } \mu_{1} \leq \mu_{2}, \\
\left(\frac{\mu_{1}}{2 \mu_{2}}, 1-\frac{\mu_{1}}{2 \mu_{2}}\right) & \text { if } \mu_{1}>\mu_{2},\end{cases} \\
& \mathbf{w}=\left(\min \left(\mu_{1}, \mu_{2}\right), 1-\min \left(\mu_{1}, \mu_{2}\right)\right) .
\end{aligned}
$$


We distinguish three cases:

(a) If $\mu_{1}<\mu_{2}$, then

$$
\begin{aligned}
& U_{P}\left(p_{1}, w_{1}\right)=\min \left(1-\frac{\mu_{2}}{2 \mu_{1}}, \mu_{1}\right)=\mu_{1}, \\
& U_{P}\left(p_{2}, w_{1}\right)=2 \frac{\mu_{2}}{2 \mu_{1}} \mu_{1}=\mu_{2} .
\end{aligned}
$$

(b) If $\mu_{1}=\mu_{2}$, then

$$
\begin{aligned}
& U_{P}\left(p_{1}, w_{1}\right)=\min \left(0.5, \mu_{1}\right)=\mu_{1}, \\
& U_{P}\left(p_{2}, w_{1}\right)=\min \left(0.5, \mu_{2}\right)=\mu_{2} .
\end{aligned}
$$

(c) If $\mu_{1}>\mu_{2}$, then

$$
\begin{aligned}
& U_{P}\left(p_{1}, w_{1}\right)=2 \frac{\mu_{1}}{2 \mu_{2}} \mu_{2}=\mu_{1}, \\
& U_{P}\left(p_{2}, w_{1}\right)=\min \left(1-\frac{\mu_{1}}{2 \mu_{2}}, \mu_{2}\right)=\mu_{2} .
\end{aligned}
$$

(2) If $\mu_{1}+\mu_{2}<1$ and $\max \left(\mu_{1}, \mu_{2}\right) \geq 2 \min \left(\mu_{1}, \mu_{2}\right)(1-$ $\left.\min \left(\mu_{1}, \mu_{2}\right)\right)$, then notice that the case $\min \left(\mu_{1}, \mu_{2}\right)=1$ is not possible. Moreover, we have

$$
\frac{\max \left(\mu_{1}, \mu_{2}\right)}{2\left(1-\min \left(\mu_{1}, \mu_{2}\right)\right)} \geq \min \left(\mu_{1}, \mu_{2}\right) .
$$

On the other hand, given that $\max \left(\mu_{1}, \mu_{2}\right)<1-\min \left(\mu_{1}, \mu_{2}\right)$, we get

$$
\frac{\max \left(\mu_{1}, \mu_{2}\right)}{2\left(1-\min \left(\mu_{1}, \mu_{2}\right)\right)}<0.5
$$

and, consequently, $\min \left(\mu_{1}, \mu_{2}\right)<0.5$. Now consider the following weighting vectors:

$$
\begin{aligned}
& \mathbf{p}= \begin{cases}\left(\mu_{1}, 1-\mu_{1}\right) & \text { if } \mu_{1} \leq \mu_{2}, \\
\left(1-\mu_{2}, \mu_{2}\right) & \text { if } \mu_{1}>\mu_{2},\end{cases} \\
& \mathbf{w} \\
& =\left(\frac{\max \left(\mu_{1}, \mu_{2}\right)}{2\left(1-\min \left(\mu_{1}, \mu_{2}\right)\right)}, 1-\frac{\max \left(\mu_{1}, \mu_{2}\right)}{2\left(1-\min \left(\mu_{1}, \mu_{2}\right)\right)}\right) .
\end{aligned}
$$

We distinguish two cases:

(a) If $\mu_{1} \leq \mu_{2}$, then

$$
\begin{aligned}
& U_{P}\left(p_{1}, w_{1}\right)=\min \left(\mu_{1}, \frac{\mu_{2}}{2\left(1-\mu_{1}\right)}\right)=\mu_{1}, \\
& U_{P}\left(p_{2}, w_{1}\right)=2\left(1-\mu_{1}\right) \frac{\mu_{2}}{2\left(1-\mu_{1}\right)}=\mu_{2} .
\end{aligned}
$$

(b) If $\mu_{1}>\mu_{2}$, then

$$
\begin{aligned}
& U_{P}\left(p_{1}, w_{1}\right)=2\left(1-\mu_{2}\right) \frac{\mu_{1}}{2\left(1-\mu_{2}\right)}=\mu_{1}, \\
& U_{P}\left(p_{2}, w_{1}\right)=\min \left(\mu_{2}, \frac{\mu_{1}}{2\left(1-\mu_{2}\right)}\right)=\mu_{2} .
\end{aligned}
$$

(3) If $\mu_{1}+\mu_{2} \geq 1$ and $\min \left(\mu_{1}, \mu_{2}\right)<2 \max \left(\mu_{1}, \mu_{2}\right)(1-$ $\left.\max \left(\mu_{1}, \mu_{2}\right)\right)$, then the proof of this case is similar to that of the second item in Theorem 18 .

(4) If $\mu_{1}+\mu_{2} \geq 1$ and $\min \left(\mu_{1}, \mu_{2}\right) \geq 2 \max \left(\mu_{1}, \mu_{2}\right)(1-$ $\left.\max \left(\mu_{1}, \mu_{2}\right)\right)$, then the proof of this case is similar to that of the third item in Theorem 18.

\section{Conclusion}

SUOWA operators are a useful tool for dealing with situations where combining values by using both a weighted mean and an OWA type aggregation is necessary. Given that they are Choquet integral-based operators with respect to normalized capacities, they have some natural properties such as continuity, monotonicity, idempotency, compensativeness, and homogeneity of degree 1 . For this reason, it seems interesting to analyze their behavior from different points of view. In this paper, we have shown that, in two dimensions, if we consider one of the following continuous semiuninorms: $U_{T_{\mathrm{L}}}, U_{T_{\mathrm{M}}}, U_{\widetilde{P}}$, and $U_{P}$, then any Choquet integral with respect to a normalized capacity can be expressed as a SUOWA operator associated with the chosen semiuninorm and two weighting vectors $\mathbf{p}$ and $\mathbf{w}$, which are given explicitly.

\section{Conflict of Interests}

The author declares that there is no conflict of interests regarding the publication of this paper.

\section{Acknowledgments}

The partial financial support from the Ministerio de Economía y Competitividad (Project ECO2012-32178) and the Junta de Castilla y León (Consejería de Educación, Project VA066U13) is gratefully acknowledged.

\section{References}

[1] R. R. Yager, "On ordered weighted averaging aggregation operators in multicriteria decisionmaking," IEEE Transactions on Systems, Man, and Cybernetics, vol. 18, no. 1, pp. 183-190, 1988.

[2] V. Torra, "Weighted OWA operators for synthesis of information," in Proceedings of the 5th IEEE International Conference on Fuzzy Systems, vol. 2, pp. 966-971, New Orleans, La, USA, September 1996.

[3] V. Torra, "The weighted OWA operator," International Journal of Intelligent Systems, vol. 12, no. 2, pp. 153-166, 1997.

[4] V. Torra, "Empirical analysis to determine weighted OWA orness," in Proceedings of the 4th International Conference on Information Fusion (FUSION '01), Montreal, Canada, August 2001. 
[5] V. Torra and L. Godo, "Continuous WOWA operators with application to defuzzification," in Aggregation Operators: New Trends and Applications, T. Calvo, G. Mayor, and R. Mesiar, Eds., vol. 97 of Studies in Fuzziness and Soft Computing, pp. 159-176, Physica, Heidelberg, Germany, 2002.

[6] V. Torra and Y. Narukawa, Modeling Decisions: Information Fusion and Aggregation Operators, Springer, Berlin, Germany, 2007.

[7] B. Roy, "Double pondération pour calculer une moyenne: pourquoi et comment?" RAIRO Operations Research, vol. 41, no. 2, pp. 125-139, 2007.

[8] R. R. Yager and N. Alajlan, "A generalized framework for mean aggregation: toward the modeling of cognitive aspects," Information Fusion, vol. 17, pp. 65-73, 2014.

[9] B. Llamazares, "Constructing Choquet integral-based operators that generalize weighted means and OWA operators," Information Fusion, vol. 23, pp. 131-138, 2015.

[10] B. Llamazares, "An analysis of some functions that generalizes weighted means and OWA operators," International Journal of Intelligent Systems, vol. 28, no. 4, pp. 380-393, 2013.

[11] H.-W. Liu, "Semi-uninorms and implications on a complete lattice," Fuzzy Sets and Systems, vol. 191, pp. 72-82, 2012.

[12] R. R. Yager and A. Rybalov, "Uninorm aggregation operators," Fuzzy Sets and Systems, vol. 80, no. 1, pp. 111-120, 1996.

[13] B. Llamazares, "SUOWA operators: constructing semi-uninorms and analyzing specific cases," Fuzzy Sets and Systems, 2015.

[14] G. Choquet, "Theory of capacities," Annales de l'Institut Fourier, vol. 5, pp. 131-295, 1953.

[15] T. Murofushi and M. Sugeno, "A theory of fuzzy measures: representations, the Choquet integral, and null sets," Journal of Mathematical Analysis and Applications, vol. 159, no. 2, pp. 532$549,1991$.

[16] M. Sugeno, Theory of fuzzy integrals and its applications [Ph.D. thesis], Tokyo Institute of Technology, Meguro, Japan, 1974.

[17] M. Maschler and B. Peleg, "The structure of the kernel of a cooperative game," SIAM Journal on Applied Mathematics, vol. 15, no. 3, pp. 569-604, 1967.

[18] M. Maschler, B. Peleg, and L. S. Shapley, "The kernel and bargaining set for convex games," International Journal of Game Theory, vol. 1, no. 1, pp. 73-93, 1971.

[19] D. Denneberg, Non-Additive Measures and Integral, Kluwer Academic, Dordrecht, The Netherlands, 1994.

[20] M. Grabisch, J.-L. Marichal, R. Mesiar, and E. Pap, Aggregation Functions, Encyclopedia of Mathematics and its Applications, Cambridge University Press, 2009.

[21] V. Torra, "On some relationships between the WOWA operator and the Choquet integral," in Proceedings of the 7th International Conference on Information Processing and Management of Uncertainty in Knowledge-Based Systems (IPMU '98), pp. 818824, Paris, France, 1998.

[22] J. Fodor, J.-L. Marichal, and M. Roubens, "Characterization of the ordered weighted averaging operators," IEEE Transactions on Fuzzy Systems, vol. 3, no. 2, pp. 236-240, 1995.

[23] M. Grabisch, "Fuzzy integral in multicriteria decision making," Fuzzy Sets and Systems, vol. 69, no. 3, pp. 279-298, 1995.

[24] M. Grabisch, "On equivalence classes of fuzzy connectives-the case of fuzzy integrals," IEEE Transactions on Fuzzy Systems, vol. 3, no. 1, pp. 96-109, 1995.
[25] M. Grabisch, T. Murofushi, and M. Sugeno, Eds., Fuzzy Measures and Integrals: Theory and Applications, vol. 40 of Studies in Fuzziness and Soft Computing, Physica, Heidelberg, Germany, 2000.

[26] M. Grabisch and C. Labreuche, "A decade of application of the Choquet and Sugeno integrals in multi-criteria decision aid," Annals of Operations Research, vol. 175, no. 1, pp. 247-286, 2010. 


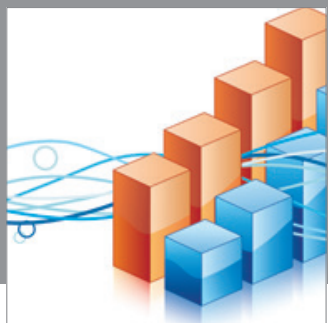

Advances in

Operations Research

mansans

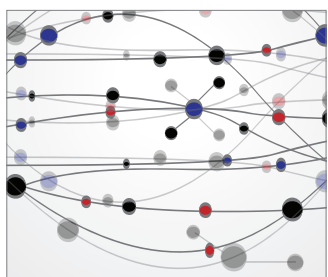

The Scientific World Journal
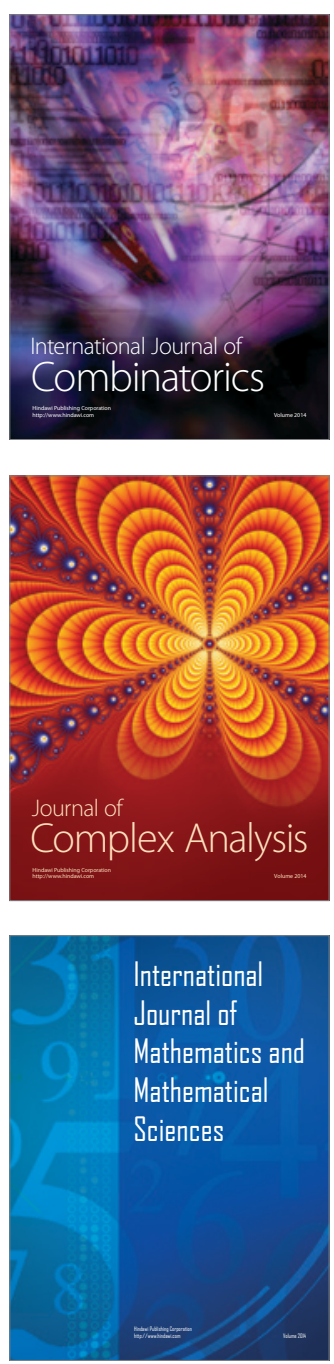
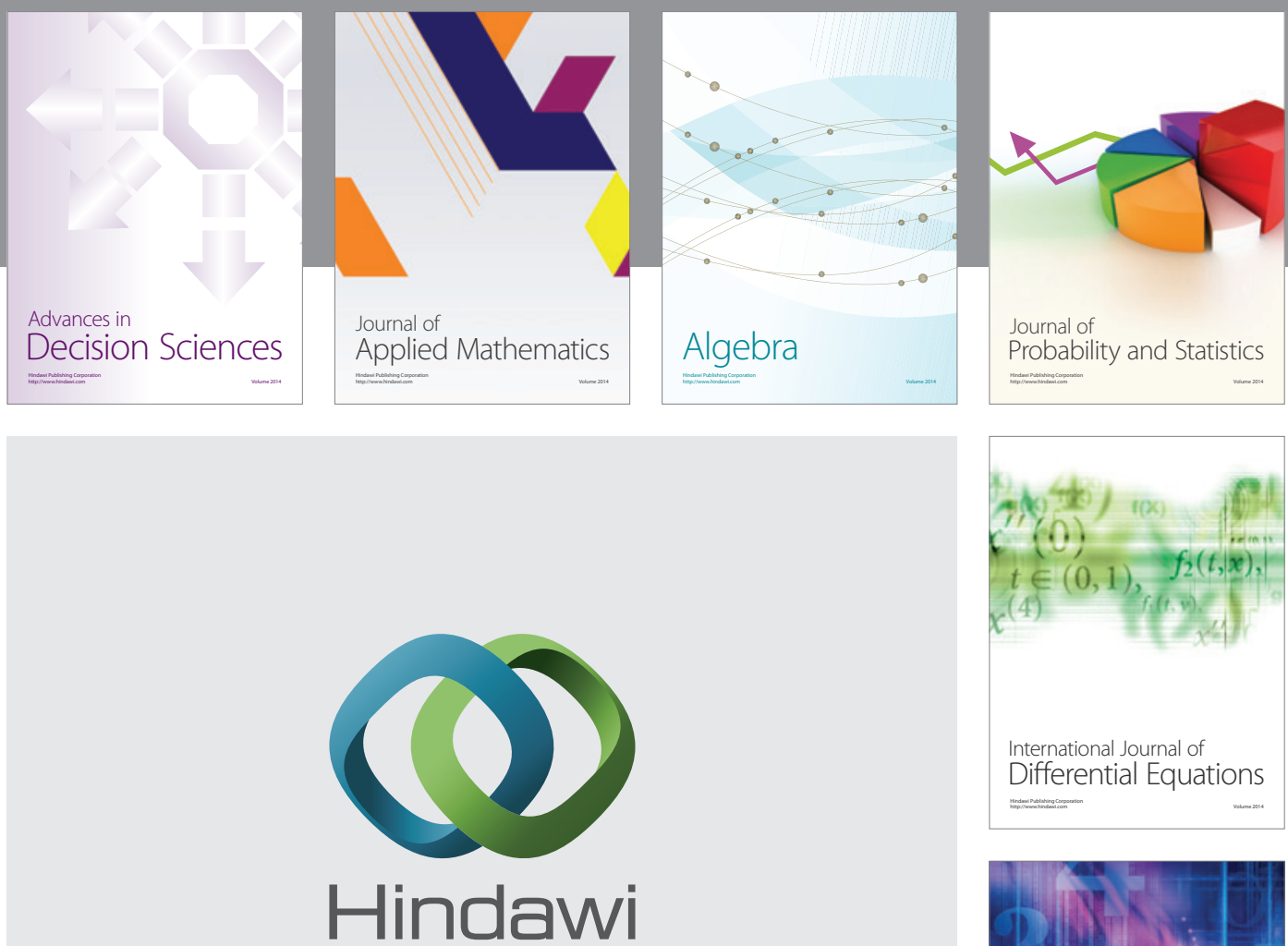

Submit your manuscripts at http://www.hindawi.com
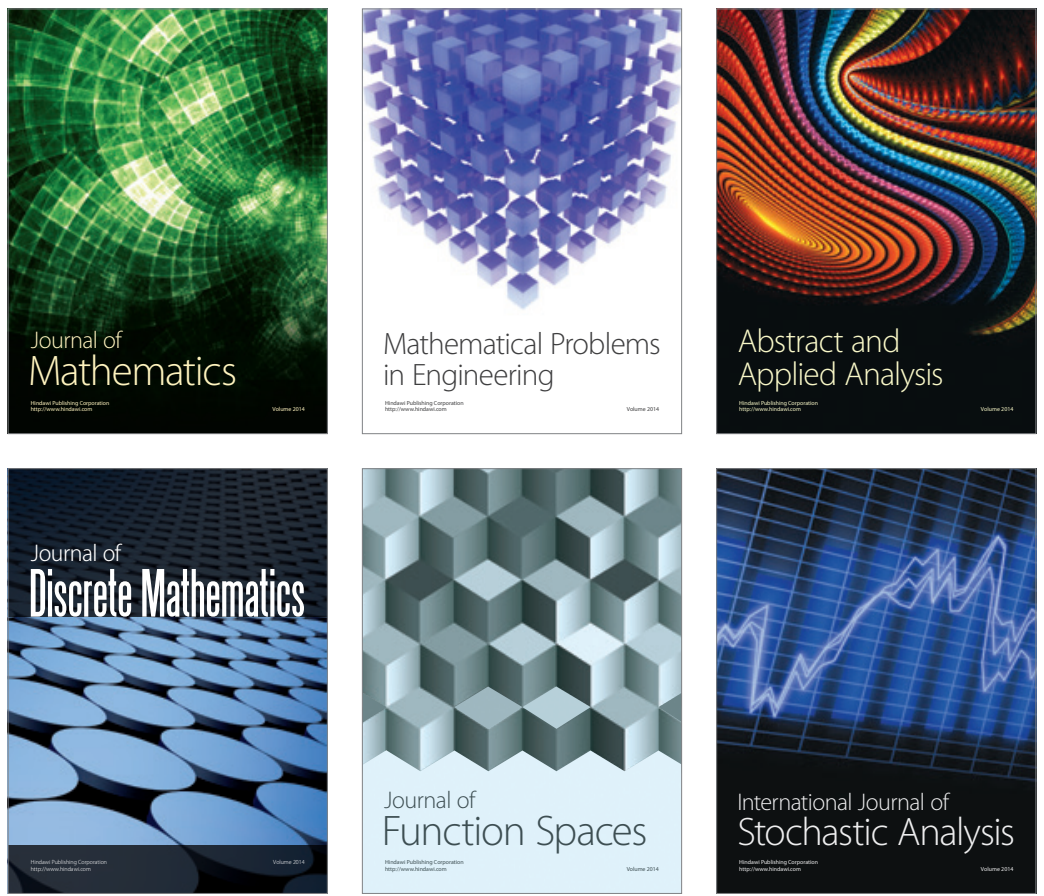

Journal of

Function Spaces

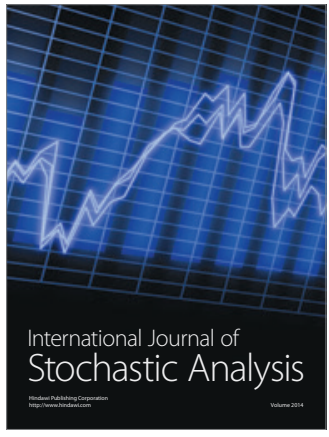

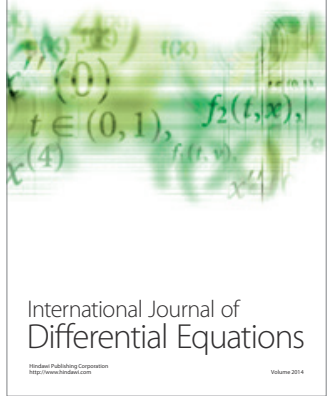
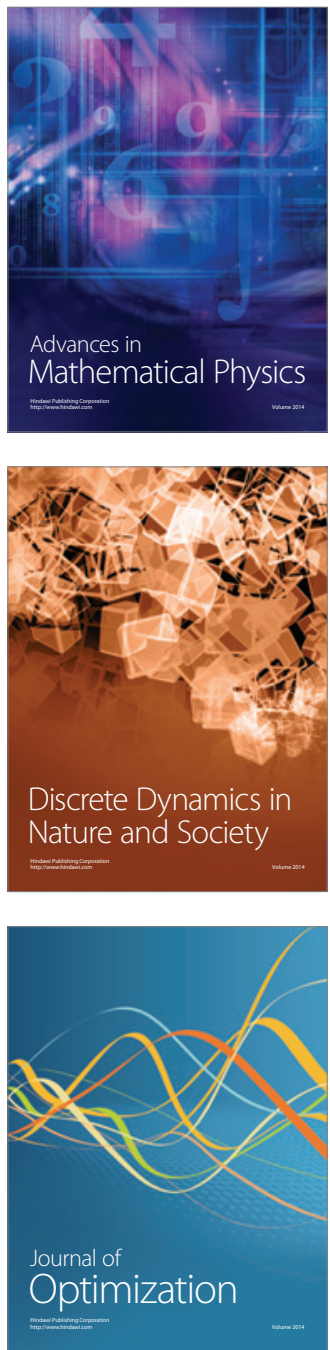